\title{
Research on the relationship between the structural properties of bedding layer in spring mattress and sleep quality
}

\author{
Liming Shen ${ }^{\mathrm{a}}$, Yu-xia Chen ${ }^{\mathrm{a}, \mathrm{b}^{*}}$, Yong Guo ${ }^{\mathrm{a}, \mathrm{b}}$, ShiLu Zhong ${ }^{\mathrm{a}}$, Fei Fang ${ }^{\mathrm{a}}$, Jing Zhao ${ }^{\mathrm{a}}$ and Tian-Yi Hu ${ }^{\mathrm{a}}$ \\ ${ }^{a}$ College of Furniture and Industrial Design, Nanjing Forestry University, Nanjing, China \\ ${ }^{b}$ College of Forest and Garden, Anhui Agricultural University, Hefei, China
}

\begin{abstract}
Mattress, as a sleep platform, its types and physical properties has an important effect on sleep quality and rest efficiency. In this paper, by subjective evaluations, analysis of sleeping behaviors and tests of depth of sleep, the relationship between characteristics of the bedding materials, the structure of mattress, sleep quality and sleep behaviors were studied. The results showed that: (1) Characteristics of the bedding materials and structure of spring mattress had a remarkable effect on sleep behaviors and sleep quality. An optimum combination of the bedding materials, the structure of mattress and its core could improve the overall comfort of mattress, thereby improving the depth of sleep and sleep quality. (2)Sleep behaviors had a close relationship with sleeping postures and sleep habits. The characteristics of sleep behaviors vary from person to person.
\end{abstract}

Keywords: bedding layer properties; sleep quality; spring mattress; movements

\section{Introduction}

Chinese people have paid much attention to the importance of bed since ancient times. In the old days, as the center of living for Chinese, bed functioned as sitting and lying furniture. Although the bed was hard, without the disturbance of artificial lighting, travel, shift and work pressure, people's sleep quality still could be guaranteed. In modern times, with life rhythm speeding up, sleep disorder problem becomes more and more serious, so people focus the problem on the mattress. Different types of mattress swarm into market and advertisers often unfounded proclaimed their mattress products as having special properties and improving sleep quality, even using diagrams showing, how their products could improve spine curvature and the contour of the mattress surface conforms to that of the body. But in fact, there is no any evidence that this mattress can improve sleep quality. Some customers spent a lot buying advanced mattress, but after using a period of time, uncomfortable symptoms (allergy, lumbar lesion and so on) appeared and they changed back to hard board. It seems to leave people an impression that Chinese suits to sleep on harder bed, not soft mattress. In fact, sleeping in harder or softer mattress was not conducive to decreasing body spine load and muscle relaxing ( Chapell , 1993 ), which also have side effect on sleep quality. Besides, some specialists suggested that person different in anthropometrical characteristics should choose different support structures Pheasant (1996 , Mahaisavariya 2002 ). Sleep quality has also a relationship with sleep posture, sleep habits and human/bed interface microenvironment and so on.

This study aimed to investigate the relationship between sleep quality of Chinese and materials and the structural properties of bedding layer in spring mattress, and to determine whether Chinese people suit harder mattress or not. To reduce external disturbing factors and get more natural sleep environment, laboratory was designed into a small apartment and the subjects was resident college student volun-

\footnotetext{
*Corresponding author. E-mail: sheherose@163.com.
} Tel.:+0086-021-85427693 
teers. Analyzed their sleep movements and evaluate their sleeping quality when they slept consecutive nights on each experimental mattress.

\section{Procedure, subjects and methods}

\subsection{Procedure}

Each subject was recorded at least 4 consecutive nights on each of the 18 mattresses, the subjects. Two weeks prior (Jacobson et al, 2007) to and during the test period they were asked to sleep in the sleep laboratory to adapt sleep laboratory conditions and maintain a constant life style without change in sleeping habits. The recording sessions usually started on Monday night and ended on Friday morning. To minimize the seasonal influence on sleep quality $(50 \%$ increase of mobility during sleep has been reported in autumn as compared to spring, Kleitman, 1963), the test took place from September to December.

\subsection{Subjects}

The subjects, participating voluntarily, were selected by personal survey. They were healthy, social interaction simple, life and sleep habits regular, not heavy snorers, and had no history or symptoms of sleep disorders. To ensure regular life style and sleep habits, they were required to still sleep on the experimental mattress in the sleep laboratory during menstrual cycles, but not recording. Medicine, tea, coffee, and other stimulating beverages were all forbidden to intake $3 \mathrm{~h}$ prior to time of going to bed. Volunteers were 4 female post graduate students with a mean age of 26years $( \pm 6.63 \mathrm{yr})$. the mean height was $159.81 \mathrm{~cm}( \pm 9.88 \mathrm{~cm})$ and mean weight $51 \mathrm{~kg}$ $( \pm 6.5 \mathrm{Kg})$.The mean body mass index was 20.2 $( \pm 3.3)$.

\subsection{Measurement of physiological variable}

Subjects slept in the sleep laboratory $(25 \pm 1$, $50 \pm 5 \%$ relative humidity) and electroencephalographic (EEG), electroencephalographic (EOG), electromyographic (EMG) and electrocardiographic (ECG) data were recorded. Two electrode $(\mathrm{O} 2 / \mathrm{T} 4$, $\mathrm{C} 4 / \mathrm{A} 1)$ and one ground electrode were placed around the cranium to record meuroelectrical activity. One channel was placed above to the outside of the right eye to recording the eye movements. Surface chest electrodes were used for recording of ECG. These leads were used to determine sleep stages. Two leads were placed on the skin of the lower limb over the anterior tibial muscle to detect submental EMG activity mirroring limb movements. This was helpful in classifying a movement as a waking period, an arousal, or spastic movement. Polysomnographic data was recorded EBNeuro, Italy micromed.

Subjects' movements were recorded in the dark using Ingra-red video cameras and observed by the technicians on monitors outside the room. Movements were differentiated according to their duration, and grouped into class I (M1), if the duration was less than 5s; classII (M2), between 5 and 10s; class III (M3) 10 and 15s and classIV (M4) more than 15s.

\subsection{Selection of mattress}

The 18 mattresses built by the same manufacturer (Nanjing Kylin Mattress). The physical characteristics (e.g. elasticity, repositioning, oscillations, and hysteresis) and firmness feature (see table 1) have been thoroughly tested according to CTBA standard.

Table 1

Overall mattress Characteristics

\begin{tabular}{llll}
\multicolumn{4}{c}{ Overall mattress Characteristics } \\
\cline { 2 - 4 } & Mean (S.D.) & & \\
\cline { 2 - 4 } & $\mathrm{D}_{\text {surface }}(\mathrm{mm})$ & $\mathrm{D}_{\text {core }}(\mathrm{mm})$ & $\mathrm{D}_{\text {bottom }}(\mathrm{mm})$ \\
\hline Mattress 01A & $24.2(3.4)$ & $66.8(4.7)$ & $15.9(1.6)$ \\
Mattress 01B & $12.4(2.3)$ & $50.4(3.4)$ & $13.8(1.4)$ \\
Mattress 01C & $22.9(3.7)$ & $58.9(4.4)$ & $12.1(0.9)$ \\
Mattress 01D & $23.1(3.6)$ & $59.1(4.0)$ & $12.4(1.3)$ \\
Mattress 01E & $19.5(2.4)$ & $71.4(5.3)$ & $19.3(1.5)$ \\
Mattress 02B & $20.4(1.8)$ & $75.8(5.2)$ & $16.3(1.7)$ \\
Mattress 03B & $17.1(1.9)$ & $55.1(4.7)$ & $15.1(1.6)$ \\
Mattress 04B & $26.9(2.4)$ & $66.5(4.6)$ & $15.7(1.9)$ \\
Mattress 05B & $26.8(1.7)$ & $86.4(4.7)$ & $18.3(2.0)$ \\
Mattress 06B & $28.5(2.2)$ & $74.4(4.2)$ & $21.4(2.3)$ \\
Mattress 07B & $22.9(1.9)$ & $82.4(4.5)$ & $22.5(1.7)$ \\
Mattress 08B & $24.4(2.3)$ & $82.3(4.9)$ & $17.7(1.4)$ \\
Mattress 09B & $26.6(2.4)$ & $81.9(4.0)$ & $17.6(0.8)$ \\
Mattress 10B & $24.8(2.1)$ & $102.5(5.8)$ & $19.3(2.2)$ \\
Mattress 11B & $26.8(1.6)$ & $93.9(4.4)$ & $21.5(1.5)$ \\
Mattress 12B & $30.6(2.6)$ & $92.6(4.3)$ & $20.6(1.8)$ \\
Mattress 13B & $28.6(1.8)$ & $90.9(4.8)$ & $20.5(1.3)$
\end{tabular}




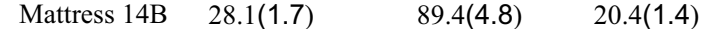

Note: The data in table is the mean and the standard deviations of overall mattress testing points.

\subsection{Questionnaires}

For each recording session the subjects had to answer two questionnaires. Prior to bedtime, the subjects reported events of relevance during the day, and noted also on a 7-position scale the level of fatigue of body parts. Next morning the subjects answered question about any external disturbance during the night, the subjective quality of sleep, level of fatigue and sleepiness, occurrence of discomfort or pain.

\subsection{Analysis}

Overnight parameters (e.g. total time in bed, total sleeping time, total number of movements per night) were obtained. The standard EEG, EOG, EMG and ECG recordings were evaluated and the predominant stage of sleep (according the manual Rechtschaffen and Kales, 1968) was assigned to the entire epoch. The first night's record was not use (Agnew wt al., 1966).

Sleep quality was assessed using sleep stages, movements and subjective estimates of sleep quality. To compare sleep quality between 18 different mattresses, the F-test for each pair of variables from all the recorded nights for the 18 mattresses conditions was used. Differences of $p<0.05$ were considered significant for all statistical analyses.

\section{Results}

The 18 mattress used in the experiment were new commercial spring mattresses, combined by five kinds of spring mattress cores and fourteen kinds of top comfort combinational layers. Mattress $01 \mathrm{C}$ and 01D were less stable, and for Mattress 09B, 10B and $12 \mathrm{~B}$ the top combinational layers were high hysteresis. All the subjects also used very thin top mattresses, filled with cotton, with the same size and specification.

The sleep structure and parameters according to the criteria of Rechtschaffen and Kale (1968) were showed in Table 1. For the eight subjects, their sleep parameters were within the normal. No significant correlation was observed between the firmness of the mattresses and the sleep parameters studied. But the sleep qualities obviously were affected by stability and hysteresis. When subjects slept on the unstable or high hysteresis mattresses (e.g. Mattress 01C and 01D with poor stability, Mattress 09B and 10B with high hysteresis), the percentages of the deep sleep $(\mathrm{S} 3+\mathrm{S} 4) \quad\left(\mathrm{M}_{01 \mathrm{C}}=15.4 \%, \quad \mathrm{SD}_{01 \mathrm{C}}=4.7, \quad \mathrm{M}_{01 \mathrm{D}}=14.8 \%\right.$, $\mathrm{SD}_{01 \mathrm{D}}=4.2, \mathrm{M}_{09 \mathrm{~B}}=15.6 \%, \mathrm{SD}_{02 \mathrm{~B}}=4.8, \mathrm{M}_{10 \mathrm{~B}}=15.4 \%$, $\left.\mathrm{SD}_{10 \mathrm{~B}}=4.3\right)$ and sleep efficiency $\left(\mathrm{M}_{01 \mathrm{C}}=94.8 \%\right.$, $\mathrm{M}_{01 \mathrm{D}}=94.6 \%, \mathrm{M}_{09 \mathrm{~B}}=95.1 \%, \mathrm{M}_{10 \mathrm{~B}}=95.0 \%$ ) were lower than those for other mattresses. Sleep efficiency, the ratio of total sleep time to time spent in bed, signifies

Table 2

Average percent sleep stage composition and the standard deviations of 8 subjects on 18 mattresses

\begin{tabular}{|c|c|c|c|c|c|c|c|c|c|}
\hline \multirow[t]{2}{*}{ Parameter } & \multicolumn{9}{|c|}{ Mean (S.D.) } \\
\hline & $01 \mathrm{~A}$ & 01B & $01 \mathrm{C}$ & $01 \mathrm{D}$ & $01 \mathrm{E}$ & 02B & 03B & 04B & 05B \\
\hline Sleep latency (\%) & $2.5(3.7)$ & $3.5(4.2)$ & $3.5(4.6)$ & $3.7(4.0)$ & $2.4(3.5)$ & $2.6(3.8)$ & $2.4(3.3)$ & $2.7(4.1)$ & $2.6(4.5)$ \\
\hline Sleep efficiency (\%) & $96.3(8.7)$ & $95.1(7.4)$ & $94.8(11.2)$ & $94.6(9.2)$ & $96.1(8.7)$ & $96.3(8.3)$ & $95.9(6.0)$ & $95.8(10.7)$ & $95.7(10.8)$ \\
\hline Awake after sleep onset (\%) & $2.4(1.3)$ & $2.5(1.2)$ & $2.8(1.2)$ & $3.1(1.5)$ & $2.1(1.1)$ & $2.1(1.2)$ & $2.3(1.3)$ & $2.2(1.3)$ & $2.2(1.4)$ \\
\hline step 1 (\%of sleep period time) & $13.0(6.8)$ & $13.3(7.4)$ & $15.5(7.2)$ & $16.4(6.9)$ & $10.1(6.5)$ & $12.7(7.5)$ & $13.7(7.1)$ & $13.4(6.9)$ & $11.2(6.2)$ \\
\hline step 2 (\%of sleep period time) & $40.2(10.1)$ & $40.4(9.7)$ & $39.9(8.4)$ & $39.1(7.8)$ & $41.6(10.3)$ & $40.2(10.2)$ & $39.7(7.6)$ & $39.5(8.4)$ & $41.2(8.5)$ \\
\hline step 3 (\%of sleep period time) & $10.1(4.1)$ & $11.0(4.5)$ & $9.9(5.0)$ & $8.7(5.3)$ & $11.2(4.4)$ & $10.7(3.9)$ & $9.7(4.8)$ & $10.7(4.8)$ & $10.3(4.7)$ \\
\hline step 4 (\%of sleep period time) & $8.3(4.3)$ & $7.4(4.3)$ & $6.5(4.6)$ & $6.1(5.6)$ & $9.3(4.0)$ & $8.3(4.0)$ & $8.3(4.5)$ & $8.5(4.4)$ & $9.5(4.2)$ \\
\hline REM (\%) & $21.2(5.3)$ & $22.9(4.9)$ & $23.6(4.8)$ & $25.1(5.5)$ & $21.3(4.1)$ & $20.6(5.2)$ & $22.6(4.5)$ & $22.4(5.2)$ & $21.7(3.6)$ \\
\hline $\mathrm{S} 3+\mathrm{S} 4$ (\%of sleep period time) & $18.6(3.8)$ & $18.4(4.2)$ & $15.4(4.7)$ & $14.8(4.2)$ & $20.5(4.0)$ & $19.0(3.6)$ & 18.1(4.7) & $19.2(4.6)$ & $19.7(4.3)$ \\
\hline \multirow[t]{2}{*}{ NREM (\%) } & $71.8(9.0)$ & $72.1(8.7)$ & $71.8(9.1)$ & $70.3(6.6)$ & $72.2(7.4)$ & $71.9(7.6)$ & $71.5(6.8)$ & $72.1(7.3)$ & $72.1(7.5)$ \\
\hline & $06 \mathrm{~B}$ & 07B & $08 \mathrm{~B}$ & 09B & $10 \mathrm{~B}$ & $11 \mathrm{~B}$ & $12 \mathrm{~B}$ & $13 \mathrm{~B}$ & 14B \\
\hline Sleep latency (\%) & $2.5(3.7)$ & $2.6(3.2)$ & $2.8(4.3)$ & $3.1(4.6)$ & $2.9(4.8)$ & $2.6(3.4)$ & $2.8(3.1)$ & $2.3(4.3)$ & $2.2(3.6)$ \\
\hline Sleep efficiency (\%) & $96.0(6.3)$ & $95.6(5.8)$ & $95.8(8.8)$ & $95.1(14.3)$ & $95.0(12.4)$ & $95.7(14.7)$ & $95.3(9.8)$ & $96.1(13.6)$ & $96.7(6.3)$ \\
\hline Awake after sleep onset (\%) & $2.3(1.3)$ & $2.4(1.2)$ & $2.2(1.1)$ & $2.8(1.4)$ & $2.9(1.2)$ & $2.2(1.4)$ & $2.7(1.3)$ & $2.0(1.0)$ & $1.9(1.3)$ \\
\hline step $1(\%$ of sleep period time) & $10.5(7.3)$ & $11(6.6)$ & $14.1(6.8)$ & $16(7.6)$ & $16.8(7.5)$ & $15.7(7.9)$ & $15(7.3)$ & $9.8(7.1)$ & $9.7(7.5)$ \\
\hline step 2(\%of sleep period time) & $41.2(9.8)$ & $41.6(10.5)$ & $40.5(7.9)$ & $39.6(7.6)$ & $39.2(7.3)$ & $39.6(6.6)$ & $40.0(8.8)$ & $42.1(9.2)$ & $41.5(9.7)$ \\
\hline step 3(\%of sleep period time) & $10.5(4.2)$ & $10.2(3.7)$ & $8.9(4.9)$ & $9.4(5.3)$ & $8.6(4.6)$ & $9.7(5.1)$ & $8.2(4.6)$ & $10.5(4.2)$ & $11.4(4.8)$ \\
\hline ) & $9.4(4.6)$ & 9.3 & $.5(4.5)$ & $6.2(5.0)$ & $6.8(4.2)$ & $7.1(4.4)$ & $7.7(4.3)$ & $9.3(3.7)$ & $9.8(4.6)$ \\
\hline
\end{tabular}




\begin{tabular}{|c|c|c|c|c|c|c|c|c|c|}
\hline (o) & 2) & 20. & ) & 23 & 3) & 5) & & & \\
\hline & & & & & & & & & \\
\hline EI & $71.6(8.8)$ & $72.1(6.7)$ & $72.0(7.7)$ & $71.2(7.1)$ & $71.4(6.9)$ & $72.1(7.3)$ & $71.9(8.6)$ & $72.7(8.7)$ & $72.4(7.2)$ \\
\hline
\end{tabular}

Table 3

Average movement's composition and the standard deviations of 8 subjects on 18 mattresses

\begin{tabular}{|c|c|c|c|c|c|c|c|c|c|}
\hline \multirow[t]{2}{*}{ Parameter } & \multicolumn{9}{|l|}{ Mean (S.D.) } \\
\hline & $01 \mathrm{~A}$ & 01B & $01 \mathrm{C}$ & $01 \mathrm{D}$ & $01 \mathrm{E}$ & $02 \mathrm{~B}$ & $03 \mathrm{~B}$ & 04B & $05 \mathrm{~B}$ \\
\hline Total movement time (min) & $24.5(18.4)$ & $24.5(23.6)$ & $28.4(24.9)$ & $31.0(26.1)$ & $22.7(20.9)$ & $20.1(16.7)$ & $19.7(12.5)$ & $23.1(18.5)$ & $25.8(24.0)$ \\
\hline Number of movements per $h$ & $41(37)$ & $43(22)$ & $62(45)$ & $80(32)$ & $40(22)$ & $35(38)$ & $34(27)$ & $37(29)$ & $57(32)$ \\
\hline Number of turns & $18(8)$ & $22(6)$ & $17(12)$ & $18(13)$ & $21(6)$ & $16(7)$ & $17(6)$ & $24(7)$ & $13(8)$ \\
\hline $\begin{array}{l}\text { Total movement time } 2 \mathrm{~d} \text { sleep } \\
\text { hours (min) }\end{array}$ & $2.3(1.5)$ & $5.0(2.6)$ & $5.5(1.9)$ & $5.7(3.3)$ & $3.3(2.9)$ & $4.4(4.0)$ & $2.3(2.0)$ & $2.5(1.9)$ & $3.7(2.5)$ \\
\hline \multirow{2}{*}{$\begin{array}{l}\text { Total movement time } 5 \text { th sleep } \\
\text { hours (min) }\end{array}$} & $5.5(2.8)$ & $3.1(2.0)$ & $4.1(2.1)$ & $4.4(1.5)$ & $4.2(2.3)$ & $3.1(1.2)$ & $4.0(2.2)$ & $4.1(3.7)$ & $5.6(2.2)$ \\
\hline & $06 \mathrm{~B}$ & 07B & 08B & 09B & 10B & $11 \mathrm{~B}$ & $12 \mathrm{~B}$ & 13B & 14B \\
\hline Total movement time (min) & $24.5(17.7)$ & $21.0(16.2)$ & $25.8(20.5)$ & $15.7(14.8)$ & $15.7(13.4)$ & $25.8(21.1)$ & $23.1(19.1)$ & $27.5(23.8)$ & $29.3(25.4)$ \\
\hline Number of movements per $h$ & $47(35)$ & $38(23)$ & $53(29)$ & $22(24)$ & $29(29)$ & $48(20)$ & $44(33)$ & $62(37)$ & $55(37)$ \\
\hline Number of turns & 19(7) & $16(8)$ & $16(13)$ & $12(7)$ & $9(7)$ & $25(37)$ & $12(37)$ & 19(9) & $22(11)$ \\
\hline $\begin{array}{l}\text { Total movement time } 2 \mathrm{~d} \text { sleep } \\
\text { hours (min) }\end{array}$ & $2.9(2.1)$ & $3.3(3.0)$ & $2.4(1.7)$ & $2.1(1.3)$ & $2.0(1.7)$ & $3.8(2.4)$ & $2.6(1.6)$ & $3.6(2.0)$ & $3.1(2.8)$ \\
\hline $\begin{array}{l}\text { Total movement time } 5 \text { th sleep } \\
\text { hours (min) }\end{array}$ & $5.0(2.3)$ & $5.1(4.0)$ & $6.0(5.0)$ & $2.6(1.5)$ & $2.8(2.2)$ & $4.6(3.5)$ & $5.1(2.9)$ & $5.4(3.7)$ & $6.2(3.6)$ \\
\hline
\end{tabular}

that a subject fell asleep quickly, and did not often awaken prior to awakening in the morning. Wake after sleep on set $\left(\mathrm{M}_{01 \mathrm{C}}=2.8 \%, \mathrm{SD}_{01 \mathrm{C}}=1.2, \mathrm{M}_{01 \mathrm{D}}=3.1 \%\right.$, $\mathrm{SD}_{01 \mathrm{D}}=1.5, \quad \mathrm{M}_{09 \mathrm{~B}}=2.8 \%, \quad \mathrm{SD}_{02 \mathrm{~B}}=1.4, \quad \mathrm{M}_{10 \mathrm{~B}}=2.9 \%$, $\mathrm{SD}_{10 \mathrm{~B}}=1.2$ ) showed a tendency to be longer with unstable mattresses and high hysteresis mattresses as compared to other experimental mattresses.

An average movement's composition and the standard deviations of the eight subjects on the 18 experimental mattresses were showed in Table 2. There was no significant correlation between the firmness of the mattresses and movement parameters. But when the mattress was unstable, the number of the movements (especially movements of classl and class ) per hour, the total duration of movements and the total movement time during the second hour increased significantly (e.g. Mattress 01C and Mattress 01D), while when the mattress hysteresis was higher, the number of the movements per hour, the total duration of movements, the total number of turns and the total duration of movements during the second hour decreasing obviously (e.g. Mattress 09B and Mattress 10). Although as the sleeping time increasing, a trend toward an increasing of class IV and turns was observed, but the time distribution of movements showed no significant different when comparing the first, second and fifth recording hours. The mean time distribution of movements expressed in \% sleep pe- riod time is presented in Table 4. No significant difference among the classes was observed.

The sleep parameters and the total amount of movements varied among the subjects, but the individual movement pattern had some reproducibility from night to night, providing that the activity and the stress during the day were moderate and that the subjects did not experience discomfort or pain during the night (except sleeping on Mattress 01D, Mattress 09B and Mattress 10B). Most of the subjects reported discomfort after sleeping at several consecutive nights on Mattress 01D (five subjects), Mattress 09B (4 subjects) and Mattress 10B (6 subject).

Comparing the 18 experimental mattresses, there was significant different in the subjective sleep ratings scores (see Figure 1) and a mild trend towards that the subjective sleep quality was increasing with the mattress firmness decreasing, although most of the subjects felt a little too soft for some of the experimental mattresses in the adapted period (prior to the test). The subjective sleep ratings scores on the Mattress 01C, 01D, 09B, and 10B were lower. 


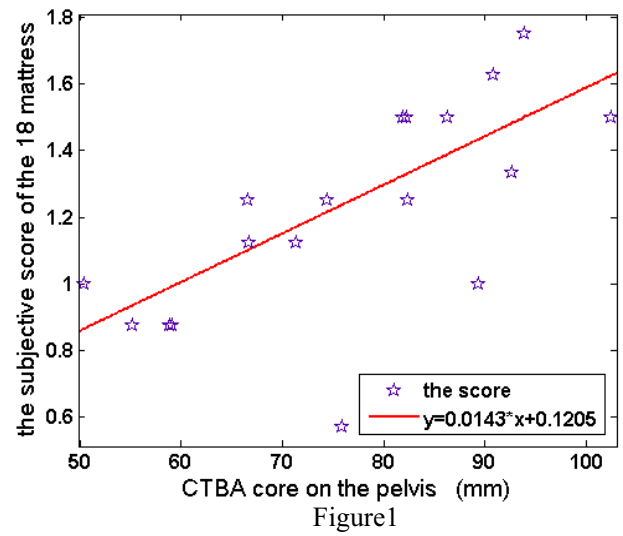

Mean of the subjective rating scores given by participants in the sleep study (overall sleep quality)

Table 4

Distribution of movements of the different class expressed in \% sleep period time

\begin{tabular}{lllll}
\hline & \multicolumn{4}{c}{ Mean (S.D.) } \\
\cline { 2 - 5 } & Class & Class & Class & Class \\
\hline Mattress 01A & $1.9(1.4)$ & $1.4(0.6)$ & $0.8(0.3)$ & $1.5(0.8)$ \\
Mattress 01B & $1.8(1.6)$ & $1.3(0.7)$ & $0.9(0.5)$ & $1.6(0.9)$ \\
Mattress 01C & $2.6(2.1)$ & $1.6(0.5)$ & $0.9(0.4)$ & $1.4(0.7)$ \\
Mattress 01D & $2.9(2.5)$ & $1.7(0.8)$ & $1.2(0.7)$ & $1.3(0.9)$ \\
Mattress 01E & $1.6(1.2)$ & $1.2(0.4)$ & $0.9(0.5)$ & $1.5(0.8)$ \\
Mattress 02B & $1.5(1.4)$ & $1.0(0.7)$ & $0.8(0.4)$ & $1.3(0.5)$ \\
Mattress 03B & $1.4(0.8)$ & $1.0(0.6)$ & $0.8(0.7)$ & $1.3(0.5)$ \\
Mattress 04B & $1.5(1.1)$ & $1.0(0.7)$ & $1.0(0.7)$ & $1.8(1.0)$ \\
Mattress 05B & $2.5(1.9)$ & $1.5(0.6)$ & $0.7(0.4)$ & $1.2(0.7)$ \\
Mattress 06B & $1.9(1.4)$ & $1.3(0.4)$ & $0.9(0.6)$ & $1.5(1.2)$ \\
Mattress 07B & $1.6(1.1)$ & $1.1(0.8)$ & $0.8(0.7)$ & $1.3(1.8)$ \\
Mattress 08B & $2.3(1.9)$ & $1.4(0.9)$ & $0.8(0.3)$ & $1.4(0.7)$ \\
Mattress 09B & $1.0(0.6)$ & $0.6(0.5)$ & $0.7(0.5)$ & $1.3(0.8)$ \\
Mattress 10B & $1.3(1.2)$ & $0.7(0.3)$ & $0.6(0.2)$ & $1.0(0.6)$ \\
Mattress 11B & $1.8(1.2)$ & $1.3(0.6)$ & $0.8(0.4)$ & $2.0(1.8)$ \\
Mattress 12B & $1.9(1.6)$ & $1.4(0.6)$ & $0.8(0.6)$ & $1.2(0.8)$ \\
Mattress 13B & $2.5(2.0)$ & $1.5(0.7)$ & $0.9(0.5)$ & $1.3(1.6)$ \\
Mattress 14B & $2.2(1.7)$ & $1.4(0.6)$ & $1.0(0.5)$ & $1.6(1.4)$ \\
\hline
\end{tabular}

\section{Discussion}

One purpose of this study was to determine the effects of mattress type on sleep quality by analyzing the sleep movements, by using a subjective mattress rating system, and through use of polysomnography.

There was no significant correlation between the mattress firmness and the sleep quality. The percentage of deep sleep and sleep efficiency were low when subjects slept on mattresses with poor stability or with top layers of high hysteresis.

As regards healthy subjects, Sucking et al. (1957) studied physiological parameters of subjects sleep on hard, medium and soft supporting surfaces (hard mattress), Bader and Engdal (2000) studied the relationship between sleep quality and bed surfaces firmness. Rosekind et al. (1976) investigated the effects of wa- terbed surfaces on sleep quality. Okamoto et al. (1997) studied about air mattress on sleep. Above studies disclosed that the mattress could not affect sleep quality. On the contrary, other studies have emphasized the fact that mattress could improve sleep quality in healthy people, e.g. Jacobson et al. (2006, 2008) studied the relationship between sleep quality, new mattress and the firmness, and Tonetti et al. (2011) studied the sleep quality and the new mattress.

If subjects were studied on an individual basis and research focused on firmness, changes in sleep quality related to characteristics of the bedding materials and springs were observed. The previous studies have not provided evidence that sleep quality differ according to the stability and hysteresis of the mattress. Our study investigated differences of sleep quality when slept on mattresses with different characteristics of the bedding materials and springs. According to our results, subjects could sleep deeply and efficiently on softer mattresses. The number of movements varied among the subjects, with some reproducibility in the movement pattern for the same subject from night to night, if there were no external disturbing factors (Bader and Engdal, 2000). The stability of the number of major body movements per night appeared to be more consistent from night to night in the same subject than was the deep or REM sleep percentage (Moses et al., 1972; Polo, 1992). Laird (1935) and Kleitman (1963) reported greater motility at the second half of the night. We did not find differences, but our results showed that the number of movements increased with the deterioration of the mattress stability, and reduced with the increase of viscoelastic.

According to our results subjective ratings correspond to the physical signal data. A study of subjective estimates of sleep quality agreed with the other objective variables (Okamoto et al., 1997). According to Kim et al.'s study (1997), bed type not only sleep quality but also subjective sleep ratings. An optimal 
mattress should facilitate higher sleep quality, and should consider individual differences in order to facilitate deep sleep (Park, 2006). When subjects slept on mattresses with poor stability or high hysteresis, the percentages of deep sleep and sleep efficiency were low, while the percentage of waking after sleep onset was high. The two factors of sleep environments affected not only sleep quality but also subjective sleep ratings.

To spring mattresses, the spring bed core was an important influencing factor of the mattress stability. The mattresses viscoelastic was related to the characteristics of the bedding materials and structure of spring mattress. Therefore, optimizing the combination of the bedding materials, the structure of mattress and its core could improve the physical properties of the mattress and the matching relation between person and the mattress, thereby promoting the quality of sleep.

\section{Conclusions}

These results showed the difference of sleep quality when subjects slept on spring mattresses with different characteristics of the bedding materials and structure. The mattresses with poor stability made people have more movements and do not favour sleep, and the high hysteresis mattresses could exert pressure on nerves and blood vessels because of prolonging immobility. Further investigations are required with the factors of the mattress stability and instability patterns. An optimum combination of the bedding materials, the structure of mattress and its core are necessary to support the spine curvature naturally and to delete unnecessary body movements, therefore those of that should facilitate higher sleep quality. The mattresses should be soft enough to avoid exerting compression on body to increasing motility and disrupted sleep.

\section{Acknowledgement}

First of all, I would like to extend my sincere gratitude to National Natural Science Foundation of China, for her research funding on my thesis. Without that, this project could not develop smoothly. I am also deeply indebted to all participants in the completion of the experiment for their direct and indirect help to me.

\section{References}

[1] T. Ishikawa, H. Ando, K. Kobayashi., a Study on Sleep Stage Estimation via Non-invasive Air Mattress Sensor [C], SICE annual conference in Fukui, 2586-2589, 2003.

[2] López-Torres, M., etc, Objective firmness, average pressure and subjective perception in mattress for the elderly [J], Applied Ergonomics, 2008, 39(1): 123-130.

[3] H. Lee, Sdjin Park, Quantitative effects of mattress type on sleep quality through polysomnography and skin temperature [J], Industrial Ergonomics, 2006,36: 934-949.

[4] F. M. Kovacs, V. Abraira, etc., Effect of Firmness of Mattress on Chronic Non-specific Low-back Pain: Rabdomised, Doubleblind, Controlled, Multicentre trial [J], The Lancet, 2003, 362 : 1599-1604.

[5] Bert H. Jacoson, Tia J. Wallace, Doug B. Smith, Grouped Comparision of Sleep Quality for New and Persongal Bedding Systems [J]. 2008, Applied Ergonomics, 39: 247-254.

[6] Zhougping Lin, Shiming Deng, A study on the thermal comfort in sleeping enviroments in the subtropics-Measuring the total insulation values for the bedding systems commonly used in the subtropics $[\mathrm{J}]$, Building and Environment, Jan, 10: 1-12, 2007

[7] Juha, M.Kortelainen, Jussi Virkkala, FFT Averaging of Multichannel BCG Signals from Bed Mattress Sensor to Improve Estimation of Heart Beat Interval [C], Conference of the IEEE EMBS, 2007, 8: 6685-6688.

[8] Ling-Ling Tsai, hau-Min Liu, Effects of Bedding Systems Selected by Manual Muscle Testing on Sleep and Sleep-related Respiratory Disturbances [J], Applied Ergonomics, 2008, 39(2): 261-270.

[9] B H. Jacobson, Tia Wallace, Hugh Gemmell, Subjective Rating of Perceived Back Pain, Stiffness and Sleep Quality Following Introduction of Medium-Firm, Journal of Chiropractic Medicine, 2006, 4: 128-134.

[10]A. Jonsson, M. Linden, M. Lindgren, Evaluation of Antidecubitus Mattresses [J], Medical Biological Engineering \& Computing, 2005, 43: 541-547.

[11] Inhyuk Moon, Sung-Jae Kang, Gyu-Seok Kim, Mu-Seong Mun, Control of air-cell mattess for preventing pressure ulcer based on approximate anthropometric model [C], ICORR 2005, 9th International Conference 2005, 28:164-167.

[12]Higashi, Y. Yuji, T. Oikawa, D. Akihiro, M. Koudabashi, A., Fujimoto, T.Masuda, Y.Tamura, Evaluation for Mattress Comfort by Using MEG [C], Conference on Neural Engineering, 2003, 22: 63-66. 